\title{
Influência do esforço amostral na riqueza de espécies de morcegos no sudeste do Brasil
}

\author{
Carlos E. L. Esbérard ${ }^{1} \&$ Helena G. Bergallo ${ }^{2}$
}

${ }^{1}$ Instituto de Biologia, Universidade Federal Rural do Estado do Rio de Janeiro. Caixa Postal 74507, 23890-000 Seropédica, Rio de Janeiro, Brasil. E-mail: cesberard@superig.com.br

${ }^{2}$ Departamento de Ecologia, Universidade do Estado do Rio de Janeiro. Rua São Francisco Xavier 524, 20559-900 Rio de Janeiro, Brasil.

\begin{abstract}
Bat richness using different sampling methodologies in southeastern Brazil. It is difficult to compare the results of bats inventories since sampling methodologies differ largely influencing the results. The objective of this study is to present three inventories of bats accomplished in similar environments in the Southeastern Brazil and to compare the different effort applied to check the bat species richness. Three methodologies were compared: I) sampling with mist nets all night, II) sampling with mist nets for six hours every night, and III) sampling with mist nets opened all night by one or two nights in each place, combined with the active search for refuges. We compared the methodologies using three different units: sampling nights, hours of effort, and number of captures. Species accumulation curves demonstrated that the addition of new species is more accelerated when the effort is of $12 \mathrm{~h}$ per night together with active searching refuges. The expected number of species given by the Chao estimator varied from 25.5 to 38 species, corresponding to 3.12 to nine species increase for each inventory. The total bat species sampled by the three methodologies reached $92 \%$ of the species recognized to occur in the south portion of Rio de Janeiro state and each inventory varied from 53.9 to $74.4 \%$ of the regional richness.
\end{abstract}

KEY WORDS. Atlantic Forest; Chiroptera; inventories; methods.

RESUMO. É difícil comparar os resultados obtidos em inventários de morcegos, pois os esforços de coleta empregados diferem grandemente, e há obviamente influência nos resultados. $O$ objetivo deste trabalho é comparar três inventários de morcegos realizados em ambientes similares no sudeste do Brasil e comparar o esforço de coleta empregado para aferir a riqueza de espécies de morcegos. Duas metodologias e três diferentes esforços de coleta foram usados: I) coleta com redes de neblina por toda a noite; II) coleta com redes por até seis horas a cada noite e III) coleta com redes armadas por 12 horas por uma ou duas noites em cada local, combinada com a busca ativa por refúgios. Nós comparamos as metodologias e os três esforços de coleta usando três unidades distintas: noites de coleta, horas de coletas e número de capturas. As curvas de acumulação de espécies obtidas demonstraram que a adição de novas espécies mostra-se mais acelerada na combinação de coletas por $12 \mathrm{~h}$ por noite e busca ativa de refúgios. O número estimado de espécies pelo estimador de Chao variou de 25,5 a 38 espécies, correspondendo de 3,12 a nove espécies a mais para cada inventário. $O$ total amostrado pelas três metodologias atingiu $92 \%$ das espécies registradas para o sul do estado do Rio de Janeiro e para cada inventário variou de 53,9 a $74,4 \%$ do total regional.

PALAVRAS-CHAVE. Chiroptera; inventário; Mata Atlântica; métodos.

A maior parte das pesquisas com morcegos do Brasil situa-se no sudeste do Brasil (Uieda \& Pedro 1996) e o estado do Rio de Janeiro é o mais bem estudado da região (Bergallo et al. 2003). Poucos autores (Simons \& Voss 1998, Bergallo et al. 2003, SAMPAIO et al. 2003) analisaram diferentes inventários de morcegos, pois é difícil comparar diferentes amostragens feitas com diferentes metodologias de coleta (Dennis \& Ruggerio 1996). Adicionalmente, entre as várias listagens disponíveis de espé- cies de morcegos há listas sabidamente incompletas, devido ao reduzido esforço de coleta, até aquelas com satisfatório esforço (Bergallo et al. 2003). Estimativas de riqueza de espécies, como as curvas de acumulação de espécies, permitem calcular quanto do total observado pode representar a fauna local (Colwell \& Coddigton 1994, Simons \& Voss 1998, SAmpaio et al. 2003), a eficiência do método adotado e a comparação dos diferentes inventários (SOBERón \& LLORENTE 1993). 
O uso de métodos estimadores de riqueza pode minimizar este problema, sendo o estimador de Chao o mais usado recentemente (CHAO 1984). Espera-se que o número de espécies raras diminua com o aumento do esforço de captura e que apenas as espécies realmente raras sejam representadas por uma ou duas capturas. Portanto, o uso do estimador de Chao permite avaliar quantas ocorrências de espécies são esperadas no local por meio do número de espécies representadas por apenas uma ou duas capturas. Quanto mais próximo do número real de espécies, maiores serão as chances de amostrar as espécies raras.

O objetivo deste trabalho foi comparar a metodologia e o esforço de coleta empregados em três inventários de morcegos realizados em ambientes similares para verificar se e quanto a metodologia e o esforço de coleta afetam a riqueza de morcegos obtida e qual seria o método mais adequado para amostrar a diversidade de Chiroptera local.

\section{MATERIAL E MÉTODOS}

A área de estudo considerada estende-se de Mangaratiba a Paraty, incluindo algumas ilhas da Enseada de Angra dos Reis, no sul do estado do Rio de Janeiro, Brasil (Fig. 1), totalizando $3800 \mathrm{~km}^{2}$. A fisionomia vegetal na área é classificada como Floresta Ombrófila Densa, com presença de restingas e manguezais (Ururahy et al. 1983). Recentemente, Furipterus horrens (Cuvier, 1828) foi registrado nesta região (Pol et al. 2003), sendo acrescentado às 43 espécies já listadas por Pereira et al. (2003) e 35 espécies de morcegos capturadas na Ilha Grande (EsBérARD et al. 2006). Estes dados corroboram com Bergallo et al. (2000) que indicam a região como área prioritária para amostragem de mamíferos devido ao baixo esforço de coleta realizado.

Foram amostrados 14 locais, com altitudes variando de 0 a $150 \mathrm{~m}$. As coletas foram agrupadas em um dos três grupos que seguem abaixo.

Grupo I. Uso de redes de neblina abertas por seis horas a cada noite, esforço total $=426$ horas. A Ilha Grande, localizada no município de Angra dos Reis, possui cerca de 19300 ha e está separada do continente por um canal de cerca de $2 \mathrm{~km}$. Suas características climáticas são similares ao sul do Estado do Rio de Janeiro, e a vegetação predominante é a Mata Atlântica, que em alguns trechos cobre montanhas que chegam a atingir mais de $1000 \mathrm{~m}$ de altitude (Maciel et al. 1984, Araujo \& OliveiRA 1988, AlHo et al. 2002). As coletas de morcegos foram realizadas no Parque Estadual da Ilha Grande, nas proximidades do Centro de Estudos Ambientais e de Desenvolvimento Sustentável da Universidade do Estado do Rio de Janeiro, localizado na Vila Dois Rios. A cada mês, três noites de coleta foram realizadas, sendo duas em pontos fixos e uma terceira em rodízio, empregando de duas a seis redes (máximo de $72 \mathrm{~m} /$ noite) abertas em bordas de mata, sobre rios ou em trilhas já existentes.

Grupo II. Uso de redes de neblina abertas por 12 horas a cada noite, esforço total $=372$ horas. A Reserva Rio das Pedras situa-se na Praia Grande no km 55 da BR 101, a cerca de $8 \mathrm{~km}$ do município de Mangaratiba e a $82 \mathrm{~km}$ do centro da Cidade

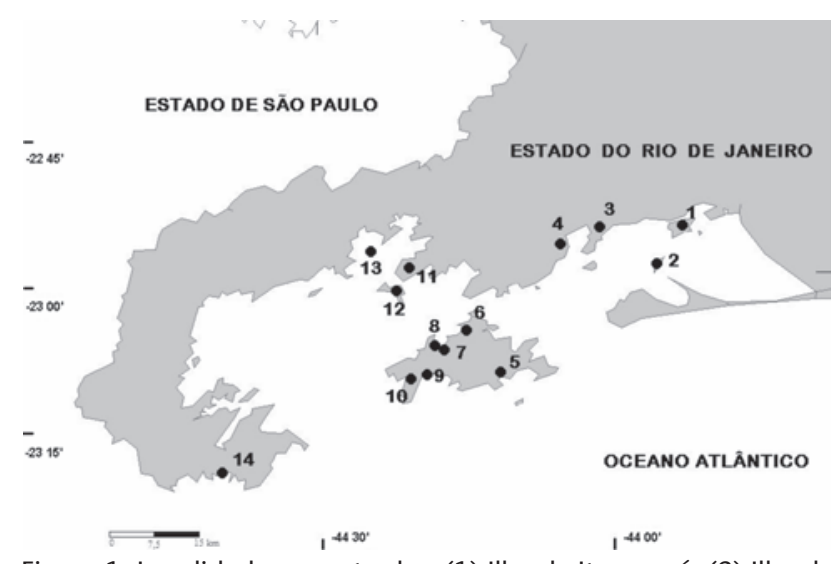

Figura 1. Localidades amostradas: (1) Ilha de Itacuruçá, (2) Ilha de Jaguanum, (3) Fazenda Portobello, (4) Reserva Rio das Pedras, (5) Vila Dois Rios, (6) Provetá, (7) Abraão, (8) Praia Preta, (10) Bananal, (9) Aventureiro, (11) Lídice, (12) Ilha da Gipóia, (13) Ilha das Palmeiras e (14) Praia do Sono.

do Rio de Janeiro $\left(22^{\circ} 59^{\prime} 26,4^{\prime \prime}\right.$ S e $\left.044^{\circ} 06^{\prime} 03,2^{\prime \prime} \mathrm{W}\right)$. A reserva possui 1361 ha, que engloba desde a região litorânea até 1150 $m$ de altitude, incluindo áreas atualmente abandonadas de cultivo de banana, matas secundárias e primárias. Limita-se a leste pela Rodovia BR-101, a norte com a Serra Grande, a oeste pela Serra Capivari e ao sul com o Município de Conceição de Jacareí. A reserva localiza-se na Bacia do Rio Grande e está inserida dentro da Área de Proteção Ambiental de Mangaratiba. Trata-se de Reserva Particular do Patrimônio Natural, mantida pelo Club Mediterraneé. Foram realizadas 31 noites de coleta, sendo uma preliminar em setembro de 1995 e as demais entre janeiro de 1997 e junho de 1998, compreendendo duas a três noites por mês. As coletas foram realizadas em todas as fases do ciclo lunar. As redes (70 m em média por noite) foram armadas antes do crepúsculo e desarmadas após o amanhecer em bordas de mata, sobre riachos e em trilhas já existentes.

Grupo III. Uso de redes de neblina e busca ativa de refúgios durante o dia, redes abertas por 12 horas, esforço total = 348 horas. Doze locais no sul do estado foram amostrados primordialmente para controle de morcegos hematófagos, armando-se média de sete redes durante duas a três noites junto aos vilarejos onde foi comprovado o ataque de Desmodus rotundus (E. Geoffroy, 1810) a seres humanos e animais domésticos. Os locais foram Ilha de Itacuruçá e Ilha de Jaguanum (município de Mangaratiba), Lidice, Ilha das Palmeiras, Ilha da Gipóia, Ilha Grande (Abraão, Provetá, Bananal, Aventureiro e Praia Preta) (município de Angra dos Reis), Praia do Sono (Município de Paraty) e Fazenda Portobello (município de Mangaratiba). As redes foram armadas em trilhas largas no interior de áreas florestadas ou na borda de fragmentos. Em todas as localidades realizamos a busca ativa por abrigos em ocos de árvores, grutas e casas abandonadas ou não, com e sem ajuda de moradores e 
foram realizadas coletas com redes de neblina por toda a noite. A procura por abrigos foi realizada no período da manhã, por quatro horas, totalizando até $24 \mathrm{~h}$ em cada localidade. Dos dez abrigos localizados, sete foram amostrados e em seis destes foram capturados morcegos (dois em telhados, um em oco de árvore, um em ponte, um em gruta, um em fenda de rocha e um em construção abandonada). Cada abrigo amostrado teve o número total de indivíduos observado quando da entrada do pesquisador, ou no caso dos telhados, no momento da saída próximo ao pôr-do-sol e este valor foi usado para calcular o estimador de Chao.

Construímos as curvas de acumulação das espécies, usando o número de noites realizadas, o número de horas de esforço e o número de capturas (Sóberon \& Llorente 1993). Moreno \& Halffter (2000) e Medellín (1993) afirmaram que o uso das unidades noites de coleta e de horas de coleta é mais adequada que o número de horas-rede, que não cresce linearmente, por isso optamos por não utilizar esta última unidade.

A riqueza esperada foi estimada empregando-se o estimador de Chao (Chao 1984, Bunge \& Fitzpatrick 1993, Colwel \& Coddington 1994). Tal cálculo leva em conta o número de espécies representadas por uma única captura e por duas capturas. A riqueza esperada é então calculada pela fórmula: $S_{\text {Chao }}$ $=\mathrm{S}_{\text {obs }}+\left(\mathrm{a}^{2} / 2 \mathrm{~b}\right)$, onde $\mathrm{S}_{\mathrm{obs}}$ é o número de espécies observadas, $\mathrm{a}$ é o número de espécies representadas por uma única captura e b é número de espécies representadas por duas capturas (Colwel \& Coddington 1994, Peterson \& Slade 1998).

Material testemunho dos animais foi depositado na coleção de referência do Projeto Morcegos Urbanos (Processo 1755/89-IBAMA). Os demais morcegos foram marcados com coleiras providas de cilindros coloridos segundo código previamente estabelecido (Esbérard \& DAEMon 1999) ou tatuados no dactilopatágio com alicate numerador e soltos no próprio local, uma a três horas após a captura.

\section{RESULTADOS}

Um total de trinta e cinco espécies foi capturado nas localidades amostradas (Tab. I). Sete famílias foram representadas: Noctilionidae (uma espécie), Emballonuridae (duas espécies), Phyllostomidae (24 espécies), Thyropteridae (uma espécie), Furipteridae (uma espécie), Vespertilionidae (quatro espécies) e Molossidae (três espécies) (Tab. I). Vinte e uma espécies foram amostradas em 71 noites e com esforço de 426 h no Grupo I, 26 espécies em 31 noites e com esforço de 372 h no Grupo II e 29 espécies em 27 noites e com esforço de 300 h com redes de neblina e mais $32 \mathrm{~h}$ de trabalho diurno no Grupo III. Diferenças significativas no total de capturas foram observadas entre os três grupos (entre os Grupos I e II $-\chi^{2}=25,41, \mathrm{df}=1, \mathrm{p}<0,000$; entre os Grupos I e III $-\chi^{2}=56,80, \mathrm{df}=1, \mathrm{p}<0,000$; entre os Grupos II e III $\left.-\chi^{2}=6,51, \mathrm{df}=1, \mathrm{p}<0,011\right)$. No entanto, diferenças significativas na riqueza de espécies foram observadas entre os Grupos I e II $\left(\chi^{2}=8,22, \mathrm{df}=1, \mathrm{p}=0,004\right)$ e entre os Grupos I e III $\left(\chi^{2}=18,35\right.$, $\mathrm{df}=1, \mathrm{p}=0,001)$, mas não entre os Grupos II e III $\left(\chi^{2}=1,77\right.$,
Tabela I. Espécies capturadas nos três inventários, realizados entre 1998 e 2003 no litoral sul do estado do Rio de Janeiro.

\begin{tabular}{|c|c|c|c|}
\hline Espécies & G1 & G2 & G3 \\
\hline \multicolumn{4}{|l|}{ Emballonuridae } \\
\hline Peropteryx macrotis (Wagner, 1843) & 0 & 0 & 1 \\
\hline Saccopteryx leptura (Schreber, 1774) & 0 & 1 & 0 \\
\hline \multicolumn{4}{|l|}{ Noctilionidae } \\
\hline Noctilio leporinus (Linnaeus, 1758) & 1 & 1 & 1 \\
\hline \multicolumn{4}{|l|}{ Phyllostomidae } \\
\hline Chrotopterus auritus (Peters, 1856) & 0 & 1 & 1 \\
\hline Lonchorhina aurita Tomes, 1863 & 0 & 1 & 1 \\
\hline Micronycteris megalotis (Gray, 1842) & 1 & 1 & 0 \\
\hline Phyllostomus hastatus (Pallas, 1767) & 1 & 0 & 1 \\
\hline Tonatia bidens (Spix, 1823) & 1 & 1 & 1 \\
\hline Trachops cirrhosus (Spix, 1823) & 1 & 1 & 1 \\
\hline Anoura caudifera (E. Geoffroy, 1818) & 1 & 1 & 1 \\
\hline Anoura geoffroyi Gray, 1853 & 0 & 0 & 1 \\
\hline Glossophaga soricina (Pallas, 1766) & 1 & 1 & 1 \\
\hline Lonchophylla mordax Thomas, 1903 & 1 & 1 & 0 \\
\hline Carollia perspicillata (Linnaeus, 1758) & 1 & 1 & 1 \\
\hline Artibeus fimbriatus Gray, 1838 & 1 & 1 & 1 \\
\hline Artibeus jamaicensis Leach, 1821 & 1 & 1 & 1 \\
\hline Artibeus lituratus (Olfers, 1818) & 1 & 1 & 1 \\
\hline Artibeus obscurus Schinz, 1821 & 1 & 1 & 1 \\
\hline Chiroderma doriae Thomas, 1891 & 1 & 1 & 1 \\
\hline Chiroderma villosum Peters, 1860 & 0 & 1 & 0 \\
\hline Platyrrhinus lineatus (E. Geoffroy, 1810) & 1 & 1 & 1 \\
\hline Platyrrhinus recifinus (Thomas, 1901) & 0 & 0 & 1 \\
\hline Pygoderma bilabiatum (Wagner, 1843) & 1 & 1 & 0 \\
\hline Sturnira lilium (E. Geoffroy, 1810) & 1 & 1 & 1 \\
\hline Vampyressa pusilla (Wagner, 1843) & 0 & 1 & 1 \\
\hline Desmodus rotundus (E. Geoffroy, 1810) & 1 & 1 & 1 \\
\hline Diphylla ecaudata (Spix, 1823) & 0 & 1 & 1 \\
\hline \multicolumn{4}{|l|}{ Thyropteridae } \\
\hline Thyroptera tricolor Spix, 1823 & 0 & 1 & 0 \\
\hline \multicolumn{4}{|l|}{ Furipteridae } \\
\hline Furipterus horrens (Cuvier, 1828) & 0 & 0 & 1 \\
\hline \multicolumn{4}{|l|}{ Vespertilionidae } \\
\hline Myotis albescens (E. Geoffroy, 1906) & 1 & 0 & 0 \\
\hline Myotis nigricans (Schinz, 1821) & 1 & 1 & 1 \\
\hline Myotis riparius Handley, 1960 & 0 & 1 & 1 \\
\hline Lasiurus ega (Gervais, 1856) & 0 & 0 & 1 \\
\hline \multicolumn{4}{|l|}{ Molossidae } \\
\hline Nyctinomops laticaudatus (E. Geoffroy, 1805) & 0 & 0 & 1 \\
\hline Molossus molossus (Pallas, 1766) & 1 & 0 & 1 \\
\hline Molossus rufus E. Geoffroy, 1905 & 0 & 0 & 1 \\
\hline Total & 21 & 26 & 29 \\
\hline
\end{tabular}

Revista Brasileira de Zoologia 25 (1): 67-73, March, 2008 
df $=1, p=0,183)$. Quatro espécies de morcegos só foram amostradas em seus refúgios e só ocorreram no Grupo III: Peropteryx macrotis (Wagner, 1843) capturado entre rochas, Molosus molossus (Pallas, 1766) em forros das casas amostradas, Molossus rufus E. Geoffroy, 1905 em oco de palmeira e Furipterus horrens em construção abandonada. Treze espécies foram amostradas com redes nos três inventários (Tab. I). Myotis albescens (E. Geoffroy, 1906) foi exclusiva ao Grupo I, enquanto Saccopteryx leptura (Screber, 1774) e Thyroptera tricolor Spix, 1823 mostraram-se exclusivas ao Grupo II e Anoura geoffroyi Gray, 1853, Platyrrhinus recifinus (Thomas, 1901), Lasiurus ega (Gervais, 1856) e Nictynomops laticaudata (E. Geoffroy, 1805) foram exclusivas ao Grupo III.

Os valores do estimador de Chao representam acréscimos que variaram de 3,12 espécies para o Grupo I a 9 espécies para o Grupo III. Apenas com os Grupos I e II foram obtidas $80 \%$ ou mais das espécies estimadas pelo estimador de Chao. Considerando-se o total conhecido no sul do estado do Rio de Janeiro, cada inventário representou de 53,85 a 74,36\% da riqueza regional. Os três inventários em conjunto amostraram 92\% das espécies de morcegos já descritas ou coletadas no sul do estado do Rio de Janeiro (Tab. II).

A composição de espécies nos três grupos mostra diferenças, com apenas quatro das espécies representadas por mais de $10 \%$ das capturas nos Grupos I e II e três das espécies com mais de $10 \%$ das capturas no Grupo III. Em cada localidade uma espécie mostrou-se dominante, respectivamente Carollia perspicillata (Linnaeus, 1758), Artibeus lituratus (Olfers, 1818) e Artibeus fimbriatus Gray, 1838.

As curvas de acumulação de espécies demonstram que a adição de novas espécies mostra-se mais acelerada no Grupo III por hora de coleta (Fig. 2), noite de coleta (Fig. 3) e captura (Fig. 4). A curva de acumulação de espécies aparentemente estabiliza-se a partir de 700 capturas para o Grupo II e não se estabiliza para os Grupos I e III (Fig. 4).

\section{DISCUSSÃO}

Os três inventários realizados totalizaram 35 espécies em 127 noites e obtiveram 50,7\% das 71 espécies citadas para o Estado do Rio de Janeiro (Esbérard \& BERgallo 2005). O somatório
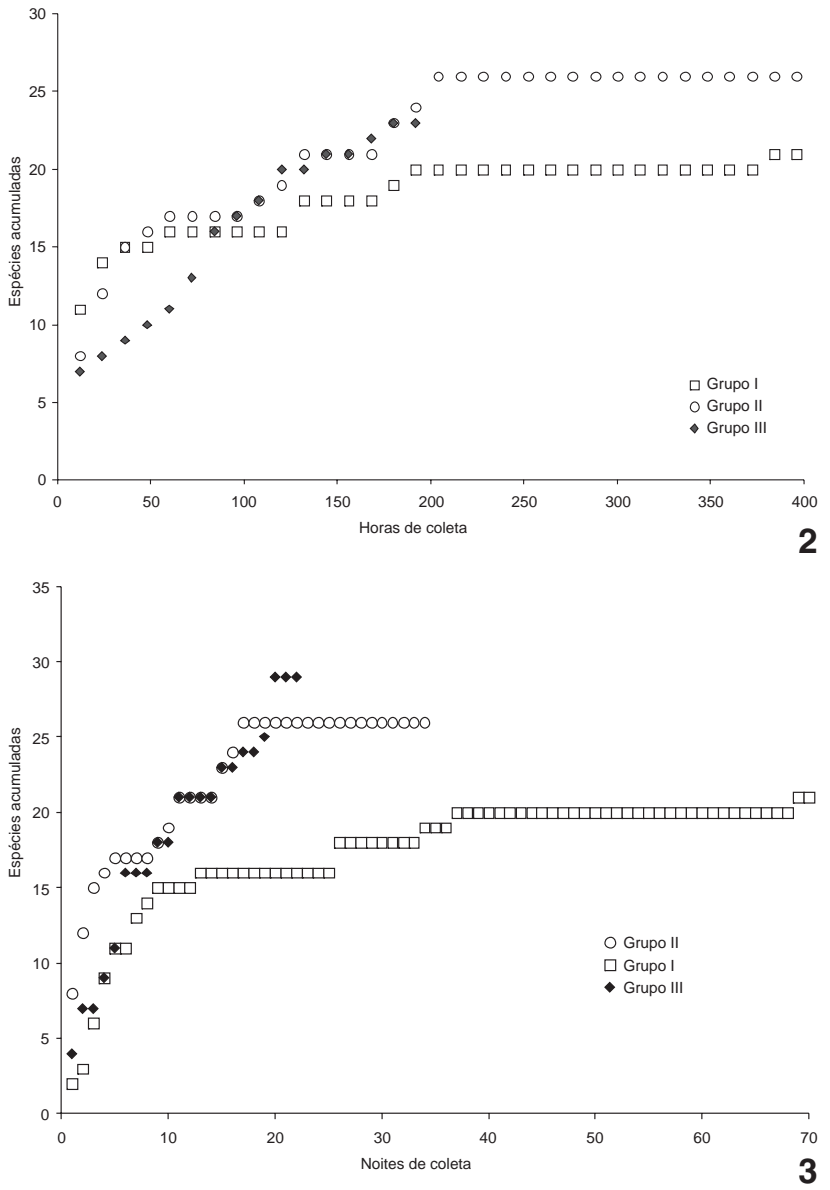

Figuras 2-3. Curvas de acumulação de espécies por horas de coleta (2) e por noites de coleta (3) para os três inventários no sul do estado do Rio de Janeiro.

dos três delineamentos, que resultou em 92\% das espécies para o sul do estado do Rio de Janeiro, mostrou-se superior ao patamar de $90 \%$ das espécies esperadas, que foi considerado satisfatório por MORENo \& HaLfFTer (2000) e SAMPAio et al. (2003)

Tabela II. Comparação entre as metodologias de amostragem usados no sul do estado do Rio de Janeiro. Completa - porcentagem do total estimado pelo Chao para amostragem total; Esperado - porcentagem do total de espécies confirmadas no sul do Estado do Rio de Janeiro. $\left(^{*}\right)$ Número de espécies encontrado no sul do Estado do Rio de Janeiro.

\begin{tabular}{lcccc}
\hline & G 1 & G 2 & G3 & Total \\
\hline Total de noites & 71 & 31 & 25 & 127 \\
Total de horas de trabalho & 426 & 372 & 348 & 1146 \\
Total de capturas & 954 & 1079 & 739 & 2772 \\
Riqueza de espécies total & 21 & 26 & $36(39)^{*}$ \\
Número estimado de espécies (Chao) & 25,5 & 30,5 & 33,5 & 39,1 \\
Completa (\% do total estimado pelo Chao) & 82,4 & 85,3 & 66,7 & 74,4 \\
Esperado (\% do total de espécies do sul do Estado) & 53,9 & & 92,3 \\
\hline
\end{tabular}

Revista Brasileira de Zoologia 25 (1): 67-73, March, 2008 

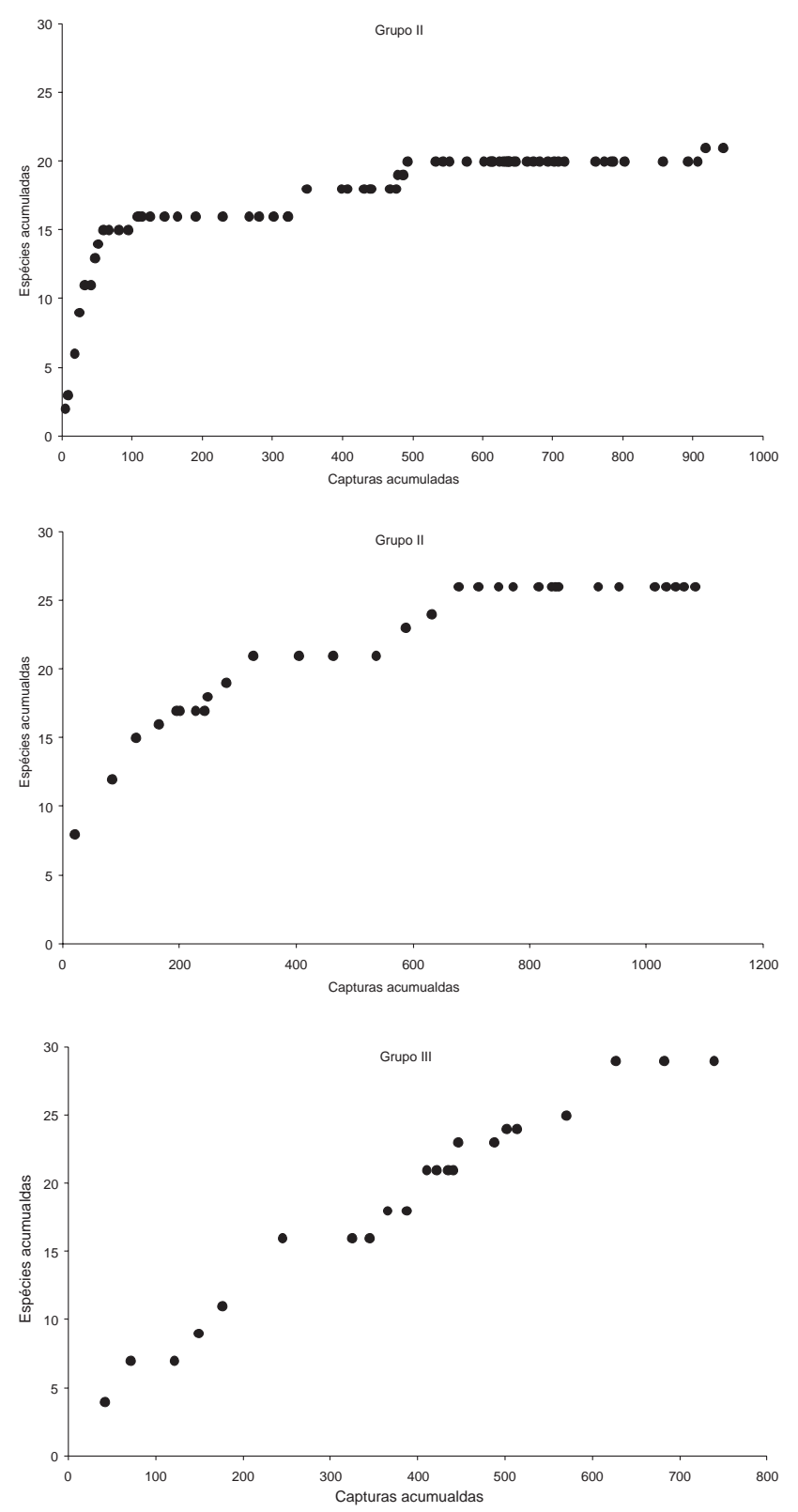

Figura 4. Curvas de acumulação de espécies por capturas para os três inventários no sul do estado do Rio de Janeiro.

ao amostrarem outras regiões. Moreno \& HalfFter (2000) estimaram que seriam necessárias de cinco a 18 noites (15 a 54 horas de coleta) para amostrar $90 \%$ da fauna de Mormoopidae e Phyllostomidae de uma pequena área homogênea e que 18 noites não foram suficientes para amostrar uma área heterogênea. SAMPAIO et al. (2003) calcularam que em 29900 horas-rede com redes no dossel e sub-bosque amostraram cerca de $95 \%$ da fauna de morcegos em Manaus, Amazonas e concluíram que eram necessárias 2000 capturas para amostrar 75\% da fauna de morcegos e mais de 6700 para atingir 95\%. Utilizando três diferentes métodos (redes no dossel, redes no sub-bosque e procura em abrigos) a estimativa atingiu cerca de $77 \%$ das espécies esperadas em Paracou, Guiana (Simons \& Voss 1998).

A soma dos três grupos resultou em mais de $92 \%$ das espécies conhecidas regionalmente, mas todos os inventários isoladamente apresentaram-se incompletos, com menos de 75\% do total regional. A estimativa de espécies pelo estimador de Chao no Grupo III (38 espécies) resultou em valor próximo ao total de espécies conhecido na região (39 espécies), no entanto, este se mostrou o inventário mais incompleto. A melhor proporção de espécies com o menor esforço de coleta foi obtida no Grupo III, com a realização de coletas e busca ativa de refúgios (uma espécie adicionada a cada $12 \mathrm{~h}$ de trabalho), no entanto este resultado não diferiu quanto à riqueza quando comparado com o Grupo II, em que o uso de redes abertas por toda a noite resultou na adição de uma espécie a cada 14,3 h de trabalho. A menor produtividade foi obtida pelo Grupo I - coletas realizadas com redes abertas por até seis horas a cada noite - uma espécie a cada 20,3 h de trabalho. A escolha da metodologia a ser adotada em um dado inventário depende primordialmente do número de noites de coleta a serem realizadas. Para menores períodos deve-se adotar a busca ativa de refúgios e coletas em diferentes pontos, distantes entre si. Para inventários com duração intermediária o uso de redes armadas durante toda a noite aparentemente pode ser empregado com resultado similar. Amostragens com redes de neblina abertas por parte da noite é o processo menos produtivo e o que menos espécies obtém (Esbérard \& Bergallo 2006).

O uso apenas de redes de neblina é o método mais empregado em inventários de morcegos em todo o Brasil (BERGallo et al. 2003, Esbérard \& Bergallo 2005), pois demanda equipes pequenas e baixo investimento. $\mathrm{O}$ uso de redes de neblina mostrase bastante funcional para espécies da Família Phyllostomidae e tende a subamostrar espécies das famílias Emballonuridae, Thyropteridae, Vespertilionidae e Molossidae (Voss \& EMONs 1996, Simons \& Voss 1998).

Quatro espécies foram amostradas apenas em seus refúgios, confirmando a necessidade de adotar diferentes métodos de coleta. A busca de refúgios demanda um conhecimento prévio do local ou maiores investimentos humanos, incluindo equipes maiores e mais longos expedientes diários de trabalho. Mesmo a combinação de dois métodos não permite a amostragem da totalidade de espécies em um período curto de tempo (Kalko et al. 1996), conforme confirmado em nossos dados.

O uso de redes necessita de elevado esforço de captura para amostrar satisfatoriamente a riqueza, sendo preconizado o mínimo de 1000 capturas para a Mata Atlântica do sudeste do Brasil (Bergallo et al. 2003). Em dois dos locais amostrados, o total obtido é próximo ao mínimo de capturas sugerido e em apenas um a curva de acumulação de espécies aparentemente estabiliza-se a partir de 700 capturas. 
Locais relativamente próximos (menos de $20 \mathrm{~km}$ ) e com fisionomia vegetal similar resultaram em diferentes abundâncias das espécies, com diferentes espécies predominando em cada local. Tal fato demonstra que para amostrar uma determinada área adequadamente, deve haver a troca constante dos pontos de coleta (veja Bergallo et al. 2003, Esbérard 2006).

Mesmo com o elevado esforço de coleta representado pela soma dos inventários não foi amostrada a totalidade de espécies de morcegos da região, onde sabemos da existência de, pelo menos, mais três espécies - Lonchophylla bokermanii Sazima, Vizotto \& Taddei, 1978, coletada na Ilha Grande (TADDEI et al. 1988), Micronycteris minuta (Peters, 1869) e Nictynomops macrotis (Gray, 1840) capturados na Ilha Grande (EsbéRARD et al. 2006) e em outras localidades não consideradas nesta análise.

Com base nos resultados obtidos parece que o método de amostragem mais eficiente é a combinação de busca de refúgio e o uso de redes abertas por toda a noite em locais trocados a cada duas ou três noites. O método menos eficiente foi o uso de redes de neblina abertas por parte da noite. Entretanto, todos os três inventários não atingiram isoladamente $75 \%$ da riqueza de espécies de morcegos para a região. Somente com a combinação de vários métodos, com um esforço de longo prazo e com muitos sítios de coleta é possível amostrar satisfatoriamente a riqueza de espécies de morcegos.

\section{AGRADECIMENTOS}

Ao Club Med, Secretaria de Saúde de Angra dos Reis, PESAGRO-RIO e UERJ pelo apoio logístico. A Rui Cerqueira, Lena Geise, Cristina A.G. Nassar, André Pol e Marco A.R. Mello pelas críticas. A Daniela Dias (UFRRJ) pela identificação de parte do material testemunho do gênero Myotis e outras espécies. Esta contribuição é parte da Tese de Doutorado de C.E.L. Esbérard desenvolvida na UERJ. Trabalho desenvolvido sob licença especial para coleta do IBAMA-DF (processos 1755/89 e 4156/95-46); recebeu financiamento do Fundo de Conservação Ambiental/SMAC (para CELE - Processo 14/001.917/97 e 14/400.116/99). C. Esbérard recebeu bolsa de Pós-Doutorado do CNPq (processo 152910/2004-0) e HGB bolsa de produtividade em pesquisa do CNPq.

\section{LITERATURA CITADA}

Alho, C.J.R.; M. Schneider \& L.A. Vasconcelos. 2002. Degree of threat to the biological diversity in the Ilha Grande State Park (RJ) and guidelines for conservation. Revista Brasileira de Biologia 62 (3): 375-385.

Araujo, D. \& R. Oliveira. 1988. Reserva Biológica Estadual da Praia Sul (Ilha Grande, Estado do Rio de Janeiro): Lista preliminar de flora. Acta Botânica Brasilica 1: 83-94.

Bergallo, H.G.; L. Geise; C.R. Bonvicino; R. Cerqueira; P.S. D'andrea; C.E.L. Esberárd; F.A.S. Fernandez; C.E.V. Grelle; A.L. Peracchi; S. Siciliano \& S.M. Vaz. 2000. Mamíferos, p. 125136. In: H.G. Bergallo; C.F.D. Rocha; M.A.S. Alves \& M.V.
SLuYs (Eds). A fauna ameaçada de extinção do Estado do Rio de Janeiro. Rio de Janeiro, Editora da UERJ, 166p.

Bergallo, H.G.; C.E.L. Esbérard; M.A.R. Mello; V. Lins; R. Mangolin; G.G. S. Melo \& M. Baptista. 2003. Bat Sampling in Atlantic Forest: How much should the minimum effort be? Biotropica 35 (2): 278-288.

Bunge, J. \& M. Fitzpatrick. 1993. Estimating the number of species: a review. Journal of American Statistical Association 88: 364-373.

CHAO, A. 1984. Nonparametric estimation of the numbers of classes in a population. Scandinavian Journal of Statistics 11: $265-270$.

Colwell, R.K. \& J.A. Coddington. 1994. Estimating terrestrial biodiversity through extrapolation. Philosophical Transactions of the Royal Society of London, Series B 345: 101-118.

DenNIS, J.G. \& M.A. RuggIERO. 1996. Biodiversity inventory: Building as inventory at scales from local to global p. 149-156. In R.C. Szaro \& D.W. Johnston (Eds). Biodiversity in managed landscapes. New York, Oxford University Press, 778p.

EsbérARD, C.E.L. 2006 Efeito da coleta de morcegos por noites seguidas no mesmo local. Revista Brasileira de Zoologia 23 (4): 1093-1096.

Esbérard, C.E.L. \& C. DAemon. 1999. Novo método para marcação de morcegos. Chiroptera Neotropical 5 (1-2): 116-117.

Esbérard, C.E.L. \& H.G. Bergallo. 2005. Research on bats in the state of Rio de Janeiro, southeastern Brazil. Mastozoologia Neotropical 12 (2): 237-243.

Esbérard, C.E.L. \& H.G. Bergallo. 2006. Coletar morcegos por seis ou doze horas a cada noite? Revista Brasileira de Zoologia 22 (4): 1095-1098.

Esbérard, C.E.L.; T.J. Jordão-Nogueira; J.L. Luz; G. Melo; R. Mangolin; N. Jucá; D.S. Raíces; M.C. EnRici \& H.G. Bergallo. 2006. Morcegos da Ilha Grande, Angra dos Reis, RJ. Revista Brasileira de Zoociências 8 (2): 147-153.

KalKo, E.K.V.; C.O. HANDley JR \& D. Handley. 1996. Organization, diversity and long-term dynamics of a neotropical bat community, p. 503-553. In M.L. Cody \& J.A. SMAlLwood (Ed.). Long-term studies of vertebrate communities. Academic Press, 597p.

Maciel, N.C.; D.S.D. Araujo \& A. Magnanini. 1984. Reserva Estadual da Praia do Sul (Ilha Grande, Angra dos Reis, RJ): contribuição para conhecimento da fauna e flora. Boletim da Fundação Brasileira para Conservação da Natureza 19: 126-148.

MedelLín, R.A. 1993. Estructura y diversidad de una comunidad de murciélagos en el trópico húmedo mexicano, p. 333-354. In: R.A. Medellín \& G. Ceballos (Eds). Avances en el estudio de los mamíferos de México. México, Publicaciones Especiales de la Asociación Mexicana de Mastozoología, 598p.

Moreno, C.E. \& G. Halffter. 2000. Assessing the completeness of bat biodiversity inventories using species accumulation curves. Journal of Applied Ecology 37: 149-158. 
Pereira, L.G.; S.E.M. Torres; H.S. da Silva \& L.Geise. 2001. Nonvolant mammals of Ilha Grande and adjacent areas in southern Rio de Janeiro State, Brazil. Boletim do Museu Nacional, Nova Série, 459: 1-15.

Peterson, A.T. \& N.A. SLADE. 1998. Extrapolating inventory results into biodiversity estimates and the importance of stopping rules. Diversity and Distributions 4: 95-105.

Pol, A.; M.R. Nogueira \& A.L. Peracchi. 2003. Primeiro registro da família Furipteridae para o estado do Rio de Janeiro (Chiroptera, Furipteridae). Revista Brasileira de Zoologia 20 (3): 561-563.

Sampaio, E.M.; E.K.V. Kalko; E. Bernard; B. Rodriguez-Herrera \& C.O. Handery JR. 2003. A biodiversity assessment of bats (Chiroptera) in a tropical lowland rainforest of central Amazônia, including methodological and conservation considerations. Studies on Neotropical Fauna and Environment 38 (1): 17-31.

Simons, N.B. \& R.S. Voss. 1998. The mammals of Paracou, French Guiana: A neotropical lowland rainforest fauna. Part 1: bats.
Bulletin American Museum Natural History 237: 1-219. Soberón, J. \& Llorente, J. 1993. The use of species accumulation functions for the prediction species richness. Conservation Biology 7: 480-488.

Taddei, V.A.; S.A. De Souza \& J.L. Manuzzi. 1988. Notas sobre uma coleção de Lonchophylla bokermanni de Ilha Grande, Sudeste do Brasil (Mammalia; Chiroptera). Revista Brasileira de Biologia 48 (4): 851-855.

Ururahy, J.C.C.; J.E.R. Collares; M.M. Santos \& R.A.A. Barretos. 1983. Folhas 23/24 Rio de Janeiro/Vitória; geomorfologia, pedologia, vegetação e uso potencial da terra, p. 553-623. In: As regiões fitoecológicas, sua natureza e seus recursos econômicos. Estudo fitogeográfico. Rio de Janeiro, Projeto RadamBrasil. vol. 4, 780p.

Uieda, W. \& W.A. Pedro. 1996. Chiroptera in the XXI Brazilian Zoology Congress. Chiroptera Neotropical 2 (1): 41-42.

Voss, R.S. \& L.H. Emmons. 1996. Mammalian diversity in neotropical lowland rainforests: a preliminary assessment. Bulletin of the American Museum of Natural History 230: 1-115.

Received in 07.VII.2006; accepted in. 